\title{
Unified Dialectical Relations of Terms in English-Chinese Translation*
}

\author{
Huaiyu $\mathrm{Mu}$ \\ Inner Mongolia University, China \\ Jijun Wang \\ Inner Mongolia University, China
}

\begin{abstract}
The differences between English and Chinese history and culture lead to the differences in the way of thinking between the two nations, and these differences in the way of thinking naturally reflect in the languages, from which the dialectical relations between English and Chinese arises in some aspects. There is a contradictory relation which is both opposite and unified between common terms in English-Chinese translation. To study the unity of opposites in English-Chinese translation is not to reconcile the contradictions, but to face up to them. Only by knowing what the problems are can we try to find a way to solve them. This paper aims to clarify the origins and definitions of these terms, make a deep analysis to them and then conclude the necessity of their independent existence in translation strategy, method and technique, and at the same time prove that under certain conditions these terms are unified, and that the two sides, which have opposing and unified relations, can also realize the transformation from one side to the other.
\end{abstract}

Index Terms—English-Chinese translation, terms, unified dialectical relations

\section{INTRODUCTION}

In the process of English-Chinese translation, the dialectical relation of unity of opposites often exists in the terms of translation strategy, method and technique. But in order to accurately understand the dialectical relation in the terms, the concepts of these terms must first be understood clearly. However, in translation studies, there is a problem that has not attracted enough attention from the academic circles, and thus it hinders the further development of translation studies to a certain extent. This problem is the confusion of concepts in translation studies, especially in the three ones, or, translation strategy, translation method and translation technique. On the one hand, although there are many discussions on translation strategy, method and technique in academic circles, it is quite rare to make a comprehensive study of them as key elements of a methodological system, and make a thorough study of their respective connotations, relations and classification systems. On the other hand, there generally exist such problems as vague definitions, improper classifications and confusing concepts in the understanding and application of these three terms in academic circles. Due to the present confusing concepts of translation strategy, method and technique, Xiong(2014) makes a detailed study and deep analysis to the concepts of translation strategy, method and technique. In his paper, he analyses the common confusion in the two basic concepts of translation strategy, translation method and translation technique in academic circles, and puts forward that a clear distinction should be made between these three concepts, which will help to eliminate the confusion in these three basic concepts, better promote translation studies and provide some enlightenment and references to the construction of the system of the translation methodology.

Based on Xiong's translation classification, this paper aims to explore unified dialectical relations of the terms in Translation strategy, Translation method and Translation technique.

\section{The Unity of OpPosites BetweEn ForeigniZATION AND DOMESTICATION IN TRANSLATION STRATEGY}

Normalization and standardization of the use of translation terms are very important prerequisites for accurate translation and the correct use of various methods, means and techniques of translation. However, in the practice of English-Chinese translation, there are some serious phenomena, such as improper use, misunderstanding and misuse of terms.

Liu (2003) believes that terms are special words which are created and used for the purposes of accurately expressing the concepts by various subjects in their fields. Their meanings usually reflect the essential characteristics of things. Translatology, as an independent subject, has gradually formed and possessed a considerable number of specialized words to express concepts in its field in the long process of development. Each term has a strictly prescribed meaning, and the use of the terms in translation studies should also be unified and standardized. Therefore, it is extremely

\footnotetext{
* This paper is the research result of the project "The Problems and Solutions of MTI Thesis in Ethnic Minority Regions"(NO. NGHWZ201729) and the project "Research on MTI Training Mode in Ethnic Minority Regions" (NO. WYZX 2017-07).
} 
necessary to give the distinct conceptual significance of the relevant translation terms, make clear their scope of use, clarify the relevance between them, and then, consistently, a strict logical system is formed ultimately. This will not only provide theoretical guidance for translation teaching, translators' translation practice, but also greatly promote normalization and standardization of the use of terminology in translation theory research.

According to Fang (2012), translation strategy can be understood as a translator's psychological tendency for achieving some translation purposes and the sum of specific methods and means which he/she adopts in the process of translation.

Xiong (2014) believes that as a macro principle and scheme, the classification of translation strategy must be closely related to the participants in translation activities. Participants in translation activities generally include the author of the original work, the sponsor, the client of the translation activity, the translator and the recipient of the translation. In this activity, the participants at the two levels of translation activities are the author of the original work and the recipient of the translation. According to the different orientations of translators to them in translation activities, translation strategy can be divided into two categories: foreignization and domestication.

Translation is not only a process of interlingual transformation, but also the one of cultural transfer. In translation activities, translators tend to have a planned tendency towards some culture to erase or highlight the linguistic and cultural differences of exotic texts (original texts), and make a plan for the translation based on this choice, this cultureoriented plan is the choice of a translator's translation strategy.

Venuti(2004), an American translation theorist, adopted the terms domestication and foreignization to describe the two different cultural tendencies in his famous book The Translator's Invisibility: A History of Translation. That is to say, domestication and foreignization, as the translation strategy, involve the processing of the cultural content of the original language.

Domestication and foreignization are a pair of contradictions, and there is a contradictory relation between them.

The essential attribute of foreignization is the author-of-the original work-orientated. That is, the translator tries to approach the author of the original work in translation. In Schleiermacher's words, the translator "tries not to disturb the original author, but to bring the reader to the original author" (Schleiermacher, 2006, p.229). Specifically, in translation, we should try our best to retain the linguistic, literary, cultural characteristics of the original work and the exotic flavor. On the contrary, the translation of domestication "does not disturb the readers' tranquility as much as possible, lets the author approach the readers" (Schleiermacher,2006, p.229), and therefore removes the barriers of language and culture for readers, but the result is the connotation of the heterogeneous culture has been cut down, changed or even assimilated, so that the exotic features of the original work can not be seen.

Domestication adheres to the expression way of the native language in translation process, and the target language, its culture or its readers are the destination of the translation. When dealing with cultural differences, domestication puts great emphasis on use of words and expression ways with the cultural features of the target language to translate the original words and expression ways, which is more in line with the expression habits of the target language, easy to be accepted by readers and convenient to communicate.

In the process of language transformation, we often encounter various obstacles caused by the differences of language and culture, some of which are even insurmountable. At this time, the translator can only adopt domestication to incorporate his own translation into the cultural norms of the target language. It is true that domestication has many advantages, but it also shows its disadvantages. Over-domestication often causes the original work to lose its true face and form a barrier to culture, leading to "Cultural dislocation". However, excessive foreignization, ignoring the needs of the readers and the language habits of the target language, blindly pursuing the correspondence with the form of the original work will lead to the obscurity of the translation.

As contradictory parties, both of them have their own occasions and opportunities to be used independently in the process of translation, and in some cases they repel, struggle and oppose against with one another, and this opposing relation between them is absolute. But sometimes they compromise and complement each other, and realize the transformation from one side to the other under certain conditions, which just reflects the unity of the two sides. Sun(2001,p.44.)says, "When we adopt foreignization, we should pay attention to the limit with great discretion, and when it is not feasible, we must resort to domestication, that is, the two methods should be complementary to each other, embodying their own advantages" .Cai(2001, p. 41) says, "In fact, foreignization and domestication are not mutually exclusive antagonistic concepts, but mutually complementary and supportive translation strategies". No matter Mr. Sun's "mutual complement" or Mr. Cai's "mutual support", they all express the same idea, that is, the relation between the two is not entirely a tit-for-tat antagonism, and both sides of the contradiction can achieve unity under certain conditions.

Translation is not static but a kind of communication between different cultures, it is a cultural phenomenon and an endless process of interpreting symbols according to different codes. Domestication and foreignization are not absolutely binary opposites. There is no absolute translation of domestication or absolute translation of foreignization. Often there is foreignization in domestication and domestication in foreignization, the main problem which strategy is dominant in translation. "They play an irreplaceable role in the target language's culture and fulfill their respective missions. Therefore, the two translation strategies will coexist forever and play a complementary role". (Guo, 1999, p.208) 
Foreignization is beneficial to cultural transplantation. According to Professor Hua (1998), when the target language readers cannot accept foreignization translation, it is necessary to adopt domestication to translate the original work by using the means of expressions with the same or similar meanings and cultural characteristics of the target language. It can effectively explain the coexistence of foreignization and domestication in translation, which shows that the two sides are not completely opposed, but are unified in opposition. For example, Lin Yutang adopted both domestication and foreignization in his translation of Six Chapters of a Floating Life, instead of mechanically adopting only one strategy from beginning to end. Because Lin Yutang is well versed in both Eastern and Western cultures and at the same time is very skilled in English and Chinese, he has successfully combined foreignization with domestication.

\section{The Unity OF OpPosites In TRANSLATION METHOD}

Translation method is aimed at linguistic forms, including word selection, sentence structure and rhetorical devices and so on. It means that due to the objective differences between the original language and the target language, such as the different expression ways and usage habits of the two languages, the translator can adopt different ways to deal with the translation, or maintain or change the language form of the original work in the process of transformation of the languages. Therefore, translation method embodies "a general dealing approach in translation, rather than a specific and partial one" ( Chesterman, 2005, p.26) .

Xiong (2014) divides translation strategy into two categories: foreignization strategy and domestication strategy. These two strategies each contain such translation methods as:

A. Translation Methods in view of foreignization: zero translation, word-for-word translation, transliteration, literal translation. Although the four translation methods are different, they have one thing in common, that is, they are all original-author-oriented, so they belong to the translation method in view of the strategy of foreignization.

B. Translation methods in view of domestication: free translation, imitation translation, variation translation, and recreation. Although the four translation methods are different, they have one thing in common, that is, they are all thetranslation-reader-oriented, so they belong to the translation method in view of the strategy of domestication.

Among these translation methods, the relation of the dialectical unity between literal translation and free translation is more obvious.

The fundamental task of translation is to fully display the "flavor" of the original work, and the "flavor" also includes the style. A translator's duty is to be faithful to the author and to convey the original flavor accurately, but at the same time the readers of the translation should be considered because the translation is for the readers of the target language. So the translator naturally has to considerate a problem of adapting to the readers' needs as much as possible in the process of translation on the premise of being faithful to the author. The two translation methods, literary translation and free translation should be adopted on this occasion. "Literal translation is a translation that not only conveys the meaning of the original work, but also pay attention to the form and is accepted by the readers, while free translation is a translation that conveys meaning regardless of the original work's form" (Li ,2004, p.21-p.25).

Literary translation is both to be faithful to the content of the original work and to maintain the form of the original work, and is consistent with the original work in such aspects as words, sentence patterns and article structures, thus retaining the expression way, figurative image and national cultural features of the original work and making the translation produce the same effect as the original. By contrast, free translation is mainly adopted in the situations where the thinking or expression way of the original work is different from that of the translation, the translation can not maintain the linguistic form of the original, that is, the same content cannot embodied by the same expression way. On this occasion, the translator expresses the main idea of the original work in his own words from the point of meaning, paying no attention to the details, thus the metaphorical image and the characteristics of national culture of the original changing or disappearing. But free translation does not mean that the content can be deleted or added at will.

Literal translation is oriented towards the superficial meaning and form of the source language, while free translation is oriented towards the deep meaning of the source language and the form of the target language.

As the two most important translation methods in translation, literal translation and free translation exist in the unity of opposites, but they are relative concepts and not excluded from each other. No matter what kind of translation, such as the translation of words, phrases, sentences, paragraphs or articles, it may be the combination of literal translation and free translation, what is only worth considering is which method is the dominant one. "Literal translation and free translation are two aspects of a basic means in translation" ( Newmark,1988, p.45 ). Literal translation emphasizes the formal consistency between the original work and the translation, while free translation emphasizes the unity of the meaning of the original work and the translation. However, they are not always mutually exclusive. In the process of translation, sometimes only literal translation need to be adopted, sometimes only free translation need to be adopted and sometimes they need to be adopted comprehensively. Literal translation and free translation are actually a dialectical unity of contradiction and interdependence, and they are two complementary translation methods. Xu(1981) believes literal translation and free translation are a pair of terms that are interrelated, complementary, integrated and penetrated into each other in complete translation. In general, literal translation should be the basis of translation methods, because literal translation adheres to the principle of unity of content and form, which is a translation method that excludes subjectivity, avoids conservatism, is good at absorbing new means of expression and has profound social 
and cultural significance, and free translation should be a supplementary means. So they are just two translation methods used alternately according to the actual situation. Translators must be good at combining the two methods. Successful translation is a combination of literal translation and free translation.

Translation is a process of dialectical unity, a dialectical unity of faithfulness and coherence, of correct understanding and exact expression, and also of literal translation and free translation. In order to achieve the level of "faithfulness, expressiveness and elegance" in translation, translators should follow dialectical thinking, regard faithfulness and smoothness as the basic criteria of translation, comprehensively apply literal translation and free translation, correctly understand the source text and accurately express the source text in the process of translation.

\section{UNITY OF OPPOSITES IN TRANSLATION TECHNIQUE}

Translation technique refers to the specific means adopted by the translator in the process of making corresponding adjustments to the words and sentences of the translation aimed at the specific situation of the differences between the source language and the target language in translating the source language. Different adjustment means of can be adopted in terms of different situations, so there are a variety of translation techniques.

Xiong (2014) classifies translation technique into five categories, namely, addition, omission, division, combination and shift.

There is a obvious contradictory relation between addition, omission, division, and combination.

Addition refers to the addition of certain words, sentences or paragraphs into the translation according to the lexical, syntactic, semantic, rhetorical or stylistic needs of the target language, or due to the constraints of certain specific cultural norms of the target language, and the addition of words implied in the original work which are not explicitly stated as well as some general and annotative words to ensure the complete meaning of the translation, making the translation grammatically correct, semantically clear, rhetorically sound, logically acceptable and culturally appropriate. Words thus supplied must be indispensable syntactically, semantically, stylistically, and contextually. On the one hand, addition can ensure the integrity of the grammatical structure of the translation, on the other hand, it can ensure the clarity of the meaning of the translation, better expressing the original ideas and contents, or better achieving specific translation purposes.

Because of the great differences between culture and expression in Chinese and English, what is added or necessary in English may be redundant in Chinese, or vice versa, hence the principle of omission in translation. Omission refers to the removal of some irrelevant words, sentences or paragraphs from the original work that are contrary to the expression habits of the translated version according to the lexical, syntactic, semantic, rhetorical or stylistic needs of the target language, or due to the constraints of certain specific cultural norms of the target language in order to express the original idea more concisely and coherently, or better achieve specific translation purposes.

There are great differences between English and Chinese in the structure of writing. Chinese is short, concise, with emphasis on parataxis and therefore the structure of articles in Chinese is loose. English places much emphasis on hypotaxis and the clear and precise logical relationship between sentences, which makes the structure of articles in English more compact. Due to the differences between English and Chinese, it is necessary to analyze and reorganize the original sentences in translation so as to conform to the characteristics of the target language. Division refers to the division of a sentence into two or more sentences when its structure in original work is long and complex. Combination refers to the combination of two or more sentences in the original work into one sentence, or of the translation of a subject-subordinate complex sentence or a compound sentence into a short sentence or a phrase in translation..

It is not necessarily faithful to the original work not to add or omit its expression, and it is not always unfaithful to the original text to add or omit its expression. On the contrary, it is not faithful to the original text not to add or omit its expression, instead, it is faithful to the original text to add or omit its expression. The relations between them are not only opposite, but also complementary, both antagonistic and unified. As a British translator said, "As addition and subtraction are crucial to mathematics, addition and omission of words are crucial to translation ” (Zhang, 1996, p.154). The purpose of adding words is to reproduce the content and style of the original work better. The principle of "faithfulness" is not violated by adding words without adding meaning and omitting words without decreasing meaning. Admission and omission are complementary and supportive mutually. Only by grasping the opportunity and the appropriate degree of addition and omission could a translator accurately and completely translate excellent works

The above analysis of these translation techniques fully demonstrates that translation technique depends on the understanding of the original work. Understanding is the basis of expression which reflects correct understanding. In the practice of translation, we should realize that translation techniques are not omnipotent and can not solve all the problems. The only use of translation technique can not guarantee that the translation is faithful and coherent, because translation is not a simple combination of parts, and the application of translation techniques is not isolated or static, no independent understanding or expression is tenable.

\section{CONCLUSIONS}

Language is the carrier of culture, and it reflects culture. Due to the differences in geography, history and culture between China and the West, there are various differences in thinking modes between them: concrete thinking and 
abstract thinking, comprehensive thinking and analytical thinking, ontological thinking and object thinking, as well as forward thinking and converse thinking. The differences in thinking modes inevitably manifest themselves in languages, leading to the differences between English and Chinese.

Among the western translation theorists, Alexander Fraser Tytler put forward the "The three principles of translation" in On the Principles of Translation in 1792: 1. The translation should give a complete transcript of the ideas of the original work. 2. The style and manner of writing should be of the same character with that of the original. 3 . The translation should have all the ease of the original composition (Chen, 2000). In order to achieve the three goals, translators must comprehensively adopt corresponding translation strategies, methods and techniques rather than choose one from the other in isolation. And the translator must give equal consideration to the two cultures at the same time, seek a balance between the two cultures in accordance with the principle of unity of opposites, and adopt various flexible means so that the translation can not only faithfully convey the original cultural information, but also appear in a reasonable form in the target culture.

In the practice of translation, there is also the factor of readers that influences how a translator translates besides the two major factors of language and culture. The translator should also take the target readers into consideration. A good translation must be permitted by its readers. The ultimate goal of conveying the spirit and culture of the original work cannot be achieved by only faithful translation of the original work regardless of the readers' acceptance ability, and also the translation could not be a good one. Only by uniting the opposites, or the original work and the target readers, taking both into account, being faithful to the original work and giving consideration to the target readers, can the translation be considered a successful one. Therefore, When translating, translators should pay attention to the differences between English and Chinese, and adopt dialectical means, such as addition and omission, division, and combination, literal translation and free translation, foreignization and domestication and so on, to improve the quality of the translation.

\section{REFERENCES}

[1] Cai, P. (2001). Domestication should be the Main Strategy in Literary Tanslation. Chinese Translators Journal, 5, 39-41.

[2] Cheng, H.W. (2000). Foundation of Chinese-English Translation. Shanghai: Shanghai Foreign Language Education Press.

[3] Chesterman, A. (2005). Problems with Strategies. In Karoly, K.\& A. Foris (Eds.). New Trends in Translation Studies: In Honour of Kinga Klaudy. Budapest: Akademiai Kiado, 17-28.

[4] Fang, Y.L. (2012). Literal Translation and Free Translation: Discussion on Translation Method, Strategy and Dimension of Meta-theory. Shanghai Journal of Translators, 3, 16-19.

[5] Guo, J.Z. (1999). Contemporary Translation Studies in USA. Wuhan: Hubei Education Press, 208.

[6] Hua, X.F. (1998). New English-Chinese Translation Course. Wuhan: Hubei People's Press.

[7] Liu, X.F. (2003). A Study on the Formulation of Comprehensive Translation Dictionaries. In Sun, Y.C (Ed.), Paper collection on Translatology Dictionary and Translation Theory. Jinan: Shandong University Press, 142-149.

[8] L, Y.S. \& H, Z.L. (Eds.)(2004). Science Translatology. Beijing: China Translation Corporation, 21- 25.

[9] Newmark, P. (1988). A Textbook of Translation. London and New York: Prentice Hall International, 45.

[10] Schleiermacher, F. (2006). On the Different Methods of Translation. In Robinson, D. (Ed.). Western Translation Theory: From Herodotus to Nietzsche. Beijing: Foreign Language Teaching and Research Press, 227-230.

[11] Sun, Z. L. (2001). China's Literary Translation: from Domestication to Foreignization. Chinese Translators Journal, 1, $40-44$.

[12] Venuti, Lawrence. (2004). The Translator's Invisibility: A History of Translation. Shanghai: Shanghai Foreign Language Education Press.

[13] Xiong, B. (2014). Conceptual Confusion in Translation Studies. Chinese Translators Journal, 3, 82-88.

[14] Xu, C.X. (1981). Re-recognition of Literal Translation and Free Translation from the Perspective of Modern Translation Practice. Journal of Foreign Languages, 6, 30-34.

[15] Zhang, J.H. (1996). On Translation. Changsha: Hunan Education Publishing House, 154.

Huaiyu Mu was born in Hohhot, the Inner Mongolia Autonomous Region, China in 1978. He received his MA degree in Foreign Linguistics and Applied Linguistics from Inner Mongolia University, China in 2009.

He is currently a lecturer in the Foreign Languages College, Inner Mongolia University, Hohhot, China. His research interests include translation studies and American literature.

Jijun Wang was born in Hohhot, the Inner Mongolia Autonomous Region, China in 1978. He received his MA degree in Foreign Linguistics and Applied Linguistics from Inner Mongolia University, China in 2007.

$\mathrm{He}$ is currently an associate professor in the Foreign Languages College, Inner Mongolia University, Hohhot, China. His research interests include translation studies and writing. 